\title{
Predicting Postpartum Haemorrhage: A systematic review of prognostic models
}

\author{
Bethany Carr ${ }^{1}$, Maryam Jahangirifar ${ }^{1}$, Ann Nicholson ${ }^{1}$, Ben $\mathrm{Mol}^{2}$, Wentao $\mathrm{Li}^{1}$, and Sharon \\ Licqurish $^{3}$ \\ ${ }^{1}$ Monash University \\ ${ }^{2}$ Monash University Faculty of Medicine Nursing and Health Sciences \\ ${ }^{3}$ Monash Centre for Health Research and Implementation
}

July 3, 2021

\begin{abstract}
Background: Postpartum Haemorrhage (PPH) remains a leading cause of maternal mortality and morbidity worldwide, and the rate is increasing. Using a reliable predictive model could identify those at risk, support management and treatment, and improve maternal outcomes. Objectives: To systematically identify and appraise existing prognostic models for PPH and ascertain suitability for clinical use. Search strategy: MEDLINE, CINAHL, Embase, and the Cochrane Library were searched using combinations of terms and synonyms including 'postpartum haemorrhage', 'prognostic model', and 'risk factors' that were developed from a scoping review. Selection Criteria: Observational or experimental studies describing a prognostic model for risk of PPH, published in English. Data Collection and Analysis: The Critical Appraisal and Data Extraction for Systematic Reviews of Prediction Modelling Studies checklist informed data extraction and Prediction Model Risk of Bias Assessment Tool guided analysis. Main Results: 16 studies met the inclusion criteria after screening 1612 records. All studies were hospital settings from 8 different countries. Models were developed for women who experienced vaginal birth $(n=7)$, caesarean $\operatorname{birth}(n=2)$, any type of $\operatorname{birth}(n=2)$, hypertensive disorders $(n=1)$ and those with placental abnormalities $(n=4)$. All studies were at high risk of bias due to use of inappropriate analysis methods or omission of important statistical considerations or suboptimal validation. Conclusions: No existing prognostic models for PPH are ready for clinical application. Future research is needed to externally validate existing models and potentially develop a new model that is reliable and applicable to clinical practice. Funding: This study received no funding. Keywords: Postpartum haemorrhage, prognostic model, prediction tool.
\end{abstract}

\section{Predicting Postpartum Haemorrhage: A systematic review of prognostic models}

Bethany L. Carr ${ }^{1 *}$, Maryam Jahangirifar ${ }^{1}$, Ann E. Nicholson ${ }^{2}$, Wentao Li ${ }^{3}$, Ben W. Mol ${ }^{3}$, Sharon Licqurish $^{1,4}$

${ }^{1}$ School of Nursing and Midwifery, Monash University, Clayton, Victoria, Australia

${ }^{2}$ Faculty of Information Technology, Monash University, Clayton, Victoria, Australia

${ }^{3}$ Department of Obstetrics and Gynaecology, School of Medicine, Monash University, Clayton, Victoria, Australia

${ }^{4}$ Monash Centre for Health Research $\&$ Implementation, Monash Health, Clayton, Victoria, Australia

* Corresponding author at: Nursing and Midwifery, Faculty of Medicine, Nursing and Health Sciences, Monash University, 35 Rainforest Walk, Clayton Campus, Clayton VIC 3800

Ph. 61399020367 
E-mail address : bethany.carr@monash.edu

Running title: Review of models predicting PPH

\section{Hosted file}

Main Document.doc available at https://authorea.com/users/423370/articles/528816-predictingpostpartum-haemorrhage-a-systematic-review-of-prognostic-models

\section{Hosted file}

Figure 1 PRISMA_2020_flow.docx available at https://authorea.com/users/423370/articles/ 528816-predicting-postpartum-haemorrhage-a-systematic-review-of-prognostic-models 